\title{
Laparoendoscopic single-site surgery versus conventional laparoscopy for hysterectomy: a systematic review and meta- analysis
}

\author{
Evelien M. Sandberg ${ }^{1}$ Claire F. la Chapelle ${ }^{1}$ Marjolein M. van den Tweel $^{1}$ • \\ Jan W. Schoones ${ }^{2} \cdot$ Frank Willem Jansen $^{1,3}$
}

Received: 17 October 2016 / Accepted: 6 February 2017 / Published online: 29 March 2017

(c) The Author(s) 2017. This article is an open access publication

\begin{abstract}
Purpose To assess the safety and effectiveness of LESS compared to conventional hysterectomy.

Methods The systematic review and meta-analysis was performed according to the MOOSE guideline, and quality of evidence was assessed using GRADE. Different databases were searched up to 4th of August 2016. Randomized controlled trials and cohort studies comparing LESS to the conventional laparoscopic hysterectomy were considered for inclusion.

Results Of the 668 unique articles, 23 were found relevant. We investigated safety by analyzing the complication rate and found no significant differences between both groups [OR $\left.0.94(0.61,1.44), I^{2}=19 \%\right]$. We assessed effectiveness by analyzing conversion risk, postoperative pain, and patient satisfaction. For conversion rates to laparotomy, no differences were identified [OR $1.60(0.40$, $6.38), I^{2}=45 \%$ ]. In $3.5 \%$ of the cases in the LESS group, an additional port was needed during LESS. For postoperative pain scores and patient satisfaction, some of the included studies reported favorable results for LESS, but
\end{abstract}

Electronic supplementary material The online version of this article (doi:10.1007/s00404-017-4323-y) contains supplementary material, which is available to authorized users.

Frank Willem Jansen

f.w.jansen@lumc.nl

1 Department of Gynecology, Minimally Invasive Surgery, Leiden University Medical Center, PO Box 9600, 2300 RC Leiden, The Netherlands

2 Walaeus Library, Leiden University Medical Center, Leiden, The Netherlands

3 Department of Biomechanical Engineering, Delft University of Technology, Delft, The Netherlands the clinical relevance was non-significant. Concerning secondary outcomes, only a difference in operative time was found in favor of the conventional group [MD $11.3 \mathrm{~min}$ (5.45-17.17), $I^{2}=89 \%$ ]. The quality of evidence for our primary outcomes was low or very low due to the study designs and lack of power for the specified outcomes. Therefore, caution is urged when interpreting the results. Conclusion The single-port technique for benign hysterectomy is feasible, safe, and equally effective compared to the conventional technique. No clinically relevant advantages were identified, and as no data on cost effectiveness are available, there are currently not enough valid arguments to broadly implement LESS for hysterectomy.

Keywords Hysterectomy · Single-port surgery · LESS · Conventional laparoscopy

\section{Introduction}

Since the early 1990s, "minimally invasive surgery" (MIS) has been rapidly implemented into a variety of surgical disciplines. The main advantage of minimally invasive procedures is the absence of a large abdominal wound, which results in fewer wound-related complications, less postoperative pain, and a shorter hospital stay [1]. In an effort to extend these benefits, an increasing enthusiasm has emerged for the laparoendoscopic single-site surgery (LESS). In LESS, multiple laparoscopic instruments are placed through one single abdominal incision at the place of the umbilicus. The hypothesis is that single incision technique might offer advantages over the standard multiport laparoscopy as abdominal wall trauma is decreased, potentially leading to less postoperative pain and improved cosmesis [2-4]. The potential drawbacks of the single-port 
approach are a larger umbilical incision $[5,6]$ and the proximity of the instruments resulting in a technical challenge, especially for advanced surgery. It was only in 1991 that Pelosi et al. performed the first LESS hysterectomy [7], more than 20 years after the first publication on the LESS procedure in 1969 [6]. Reports have currently shown the feasibility of LESS surgery in many benign gynecologic procedures $[8,9]$. However, it remains debatable whether this new technology has added value over the existing conventional laparoscopic technique and whether it should be broadly implemented for hysterectomy.

The proportion of laparoscopic hysterectomies (LH) has significantly increased the last decades: from 3\% in 2002 to $36 \%$ in 2012 in the Netherlands [10], and similar numbers have been observed in other countries (United States [11] and Finland [12]). Regarding the proportion of hysterectomies performed using the LESS approach, no national overviews have been published on this topic so far. In some parts of the world, single-port hysterectomy seems well implemented. A retrospective single-hospital study from Korea showed for example that in 2013, $80 \%$ of their hysterectomies were LESS hysterectomies [13]. Hysterectomy in general is one of the most performed advanced surgeries in gynecology with approximately 600,000 procedures a year in the United States [11]. As a result, defining the surgical approach with the most advantages is essential. In this light, the aim of this study is to provide a systematic review and meta-analysis of the current comparative studies evaluating specifically LESS hysterectomy and conventional laparoscopy. We particularly focused on the safety and effectiveness of the two techniques.

\section{Materials and methods}

\section{Eligibility criteria, information source, search strategy}

This systematic review was conducted according to the MOOSE guidelines [14]. We identified original published studies through a search of Medline (PubMed version), EMBASE (Ovid version), Cochrane, Web of Science, Central, CINAHL, Academic Search Premier and Science Direct up to 4th of Augustus 2016 without restriction. The search terms included 'gynecology', 'hysterectomy', and all acronyms of LESS. The exact search terms are presented in supplemented material (Appendix 1). In addition, relevant studies cited in the reference lists of the selected papers were evaluated. Only comparative studies (randomized controlled trials, prospective and retrospective cohort studies) evaluating LESS versus hysterectomy for benign indications were considered for inclusion. LESS procedures had to be strictly performed through one single (umbilical) port as opposed to the conventional laparoscopic hysterectomy performed through more than one port. Studies on animals or patients aged $<18$ years were excluded as well as studies comprising endoscopic surgery with different techniques (e.g., hand- or robot-assisted, isobaric pneumoperitoneum). We also excluded descriptive review articles, surveys, technical reports, published abstracts without a full manuscript, reports from meetings, and trials with less than ten included participants per arm or 20 in total.

\section{Study selection}

Two reviewers independently screened the titles and abstracts for their relevance (ES and CC). Potentially relevant studies were obtained in full text and assessed for inclusion. We included studies wherein the effectiveness and/or safety of LESS compared to conventional laparoscopy for hysterectomy were investigated. To assess the safety of a procedure, we considered complication rates as primary outcome. Effectiveness refers to the potential success of a surgical procedure, and therefore, we considered: success rate (defined by the chance for a successful procedure without conversion to laparotomy and for the use of an additional port in the single-site group), postoperative pain scores, cosmetic outcomes, and patient satisfaction (including sexual function) as relevant primary outcomes. The following secondary perioperative outcomes were considered: operative time, intraoperative blood loss, and length of hospital stay. Although less important, these are also relevant identifiers for the effectiveness of a procedure.

Complications were defined according to the classification of the Dutch Society of Obstetrics and Gynecology and further divided into 'major complications' and 'minor complications' [15]. Major complications included: major hemorrhage or hematoma (requiring transfusion); urinary tract or bowel injury; pulmonary embolism; major anesthesia problems; vaginal cuff dehiscence; port site hernia; and re-operation. Minor complications were defined as hemorrhage (not requiring transfusion) or hematoma (with spontaneous drainage); infection to the chest, urinary tract, wound, pelvic, other, or pyrexia $38^{\circ} \mathrm{C}$; deep vein thrombosis; and other minor complication requiring treatment (including voiding dysfunction and ileus). We distinguished two types of conversion: an unintended conversion to laparotomy and the need for an additional port in the single-site group. The postoperative pain should be expressed on a self-reported scale [e.g., visual analogous scale (VAS), numerical rating scale (NRS)] [16], and for cosmetic outcomes, validated questionnaires should be used.

\section{Data extraction}

Outcome data as mentioned in the previous heading as well as study and patient characteristics were extracted 
from the included studies. These baseline findings included study design, number of included participants, country where the study was conducted, source of funding, relevant characteristics of the participants (age, body mass index, and uterine weight), description of the procedural setting, and experience of the physician. Data related to the defined outcomes were assessed for inclusion in the meta-analysis. Sensitivity analyses were performed for randomized studies and cohort studies when relevant subgroup analyses were accomplished for TLH and LAVH.

\section{Assessment of risk of bias}

The study limitations in randomized trials and observational studies were assessed using the checklists adapted from Guyatt et al. [17]: (1) random sequence generation; (2) allocation concealment; (3) blinding of participants, surgeons, and investigators; (4) attrition bias: loss to followup (5) reporting bias: selective reporting and/or missing per protocol analysis; (6) other, e.g., use of non-validated outcome measures, difference in baseline characteristics between the groups and influence of co-interventions, or differing surgical experience in the compared procedures. For the first three points of the checklist, retrospective studies were rated as 'high risk', whereas attrition bias and reporting bias were marked as 'unclear', unless there was an additional reason to judge them as 'high risk'. The quality of evidence was then rated following the Grading of Recommendations Assessment, Development, and Evaluation (GRADE) approach [18]. The quality of evidence was classified into one of four categories: high quality, moderate quality, low quality, or very low quality. We used the online GRADE program (GRADEpro Guideline Development Tool [Software], McMaster University, 2015, developed by Evidence Prime, Inc., available from gradepro. org). Any discrepancies between reviewers were addressed by an open discussion.

\section{Data/Evidence synthesis and statistical analysis}

Meta-analysis was conducted using Review Manager (Version 5.2. Copenhagen: The Nordic Cochrane Centre, The Cochrane Collaboration, 2012). For continuous data, we calculated mean differences (MDs) and standard deviations (SDs); for dichotomous data, we calculated odds ratio (OD) with their 95\% confidence intervals (CIs). When summary data were missing, e.g., only the median and range were available, data were transformed as appropriate according to the definitions described by Hozo [19]. We applied the random-effects model to combine data for meta-analysis.

\section{Results}

\section{Study selection}

Figure 1 shows the flow diagram of the literature selection for this review. The initial search yielded 668 unique references, and twenty-three studies fulfilled our inclusion criteria. Eleven studies compared LESS hysterectomy to conventional TLH [13, 20-29], eleven studies compared LESS hysterectomy to LAVH [30-40], and in one study, both procedures were included [41]. Two studies also included supra-cervical hysterectomies [20, 21]. The study by Koyanagi [42] was excluded as all data were already included in another study by the same author [40]. The selected papers were published between 2010 and 2015.

\section{Study characteristics}

The included studies on LESS hysterectomy versus conventional hysterectomy are described in detail in the tables 'characteristics of included studies' (Appendix 2). A total of 1985 women in the LESS group and 2466 women in the conventional hysterectomy were included

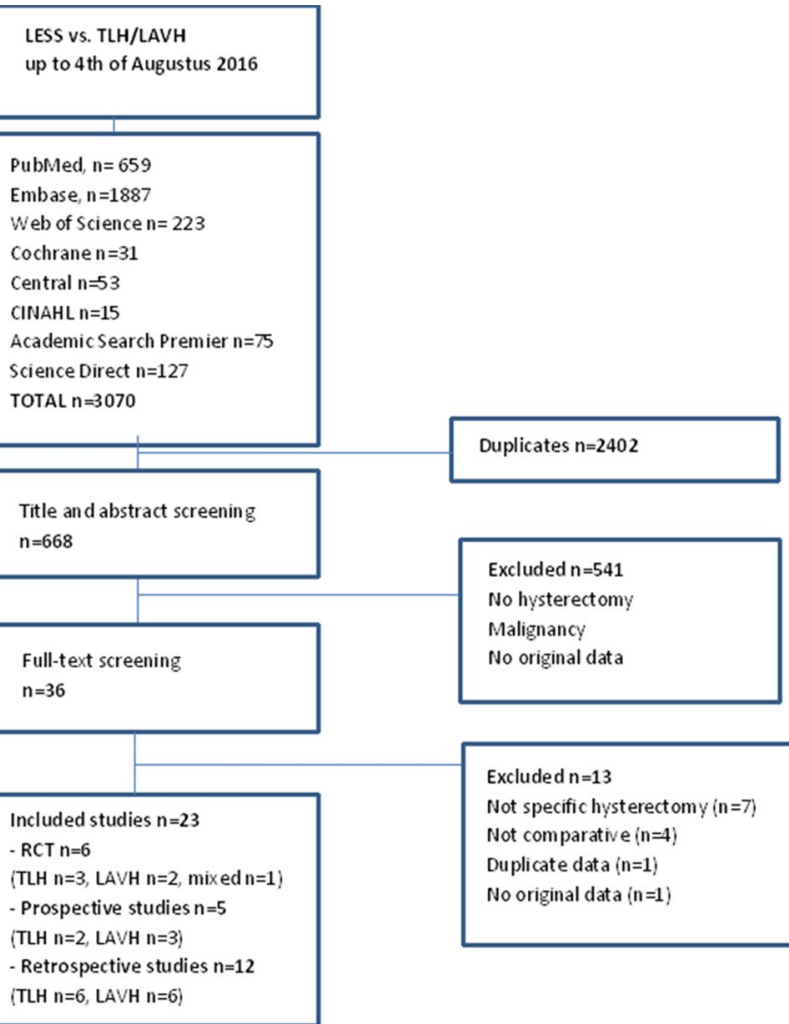

Fig. 1 Flow diagram of the literature search 
in six randomized controlled trials $[23,24,26,30,39$, 41], five prospective cohort studies [21, 27, 32, 36, 37], and 12 retrospective cohort studies [13, 20, 22, 25, 28, $29,31,33-35,38,40]$. Twenty of the studies (86.9\%) were performed in Asia (fifteen in Korea [13, 23-25, 27, $28,31,32,34-39,41]$, one in China [26], two in Japan $[29,40]$, and two in Taiwan [30, 33]), and the other three studies originated from the United States [20], Italy [22], and France [21]. Fourteen studies had a single center design [20-24, 26-30, 33, 36, 37, 39], one RCT was multi-center, and in the other eight studies, the setting was unclear [13, 25, 31, 32, 34, 35, 38, 40].

Fifteen studies stated that there was no potential conflict of interest to disclose [13, 20-27, 30-33, 35, 38], five studies reported financial support (from a grant of Samsung Medical Center [39], from a grant of Korea Health Care technology [36, 37], from Covidien [41], and from Kyung Hee University Research Fund [34]), and three studies remained unclear about their potential conflicts $[28,29,40]$.

Women in the LESS group aged between 40.3 and 53 years, their BMI ranged from 22.0 to $28.7 \mathrm{~kg} / \mathrm{m}^{2}$, and their uterine weight ranged from 105 to 642 grams. In the conventional group, the age-range of the patients, their BMI, and uterine weight varied, respectively, between 41.26 and 63 years; $22.0-28.8 \mathrm{~kg} / \mathrm{m}^{2}$ and $9-613 \mathrm{~g}$. In two studies from Lee et al., the same cohort was partially used: the smaller cohort study focused on outcomes of sexual function. We used the data from the largest cohort [37], but for analysis of the outcome 'sexual function', we extracted the data from the partial cohort [36].

\section{Risk of bias of the included studies}

A summary of risk of bias for the individual studies is depicted in Fig. 2. For the overview of GRADE findings, see Table 1.

\section{Safety: complications}

We found no differences between complication rates when comparing LESS hysterectomy to conventional hysterectomy when clustering into major complications (23 studies, OR $0.94(0.61,1.44), I^{2}=19 \%$, Fig. 3a) and minor complications (13 studies, OR 0.76 (0.46-1.27), $I^{2}=11 \%$, Fig. 3b). Sub-analysis specific for TLH and LAVH showed no difference (data not shown). None of the studies reported a port site herniation, though only one study mentioned that they had collected data on herniations [26].
Effectiveness: success rate, postoperative pain scores, cosmetic results, and patient satisfaction

Conversion to laparotomy occurred in 22 of 1835 patients (1.2\%) in the LESS group, compared to 8 of $2289(0.35 \%)$ patients in the conventional group, which was not statistically significant (total 21 studies, OR $1.60(0.40,6.38)$, $I^{2}=45 \%$, Fig. 3c). The six RCTs included and reported two conversions in both groups. For the 15 cohort studies, seventeen of the 20 conversions in the LESS group were observed in one study [13]. Reason for conversions was extensive adhesions $(n=18)$, bladder injury $(n=1)$, bladder and bowel injury $(n=1)$, retroperitoneal bleeding $(n=1)$, and unspecified $(n=9)$. When evaluating the rate of additional ports needed during LESS surgery, 48 of the 1344 (3.5\%) patients included had at least one additional port during LESS surgery versus one in the conventional group $(0.06 \%)$ [38]. Fourteen of these cases can be attributed to Fridman et al. where additional port was needed in $38 \%$ of the cases [20]. In the study by Jung et al. one patient had an additional port due to an incidental finding of an appendiceal mucinous adenoma [34].

Thirteen studies assessed the pain scores of their patients at various postoperative moments (direct after surgery up to one week) using VAS scores. Five of these studies were RCTs and one had appropriate double blinding. That specific RCT found no difference between the two groups at any of the reported moments (direct, 12, 24, and $48 \mathrm{~h}$ postoperative) [23]. The pain scores direct, 12 and $24 \mathrm{~h}$ after surgery were most frequently studied and, therefore, pooled for meta-analysis. Data that analyzed pain scores in the recovery unit, thus immediately after surgery, showed significantly lower pain scores after LESS hysterectomy compared to conventional hysterectomy ( 5 studies, MD -1.09 $(-1.66,-0.52), I^{2}=80 \%$, Fig. 4a) $[21-23,28]$. The only randomized controlled trial included in this sub-analysis showed no difference between the two groups. At $12 \mathrm{~h}$, a non-significant difference was observed ( 5 studies, MD $-0.19(-0.41,0.03), I^{2}=0 \%$, Fig. 4b). At 24 h, meta-analysis showed a significant difference between the two groups (11 studies, MD $-0.45(-0.87,-0.03), I^{2}=90 \%$, Fig. 4c) $[21,23,25,28]$. Though, the subgroup analysis including five RCTs showed non-significant results (MD -0.15 $[-0.58,0.28] . I^{2}=64 \%$ ).

Ten studies reported on data regarding analgesic use [22-25, 28, 30, 33, 38, 39, 41]. Chung et al. and Jung et al. showed that the LESS group requested significantly more (additional) analgesics, but the VAS scores revealed no difference [23, 24]. In contrast, the (rescue) analgesic requirement was significantly lower in the LESS group in four studies [22, 28, 30, 38]. Similarly, Hong et al. calculated a pain-relief score based on the amount and type of analgesic used and the effectiveness 
Fig. 2 Risk of bias summary LESS versus conventional laparoscopic hysterectomy

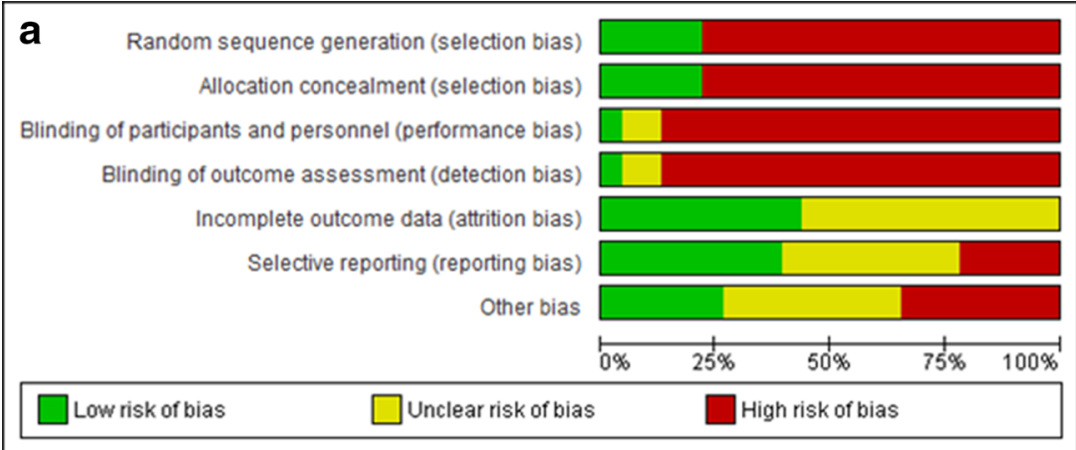

b

\begin{tabular}{|c|c|c|c|c|c|c|c|}
\hline & 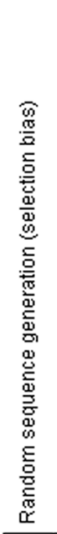 & 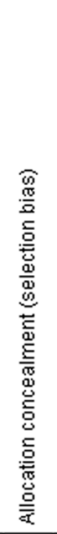 & 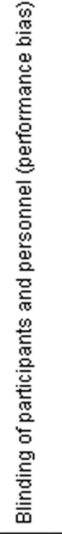 & 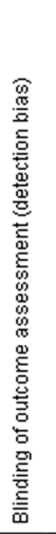 & 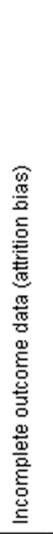 & 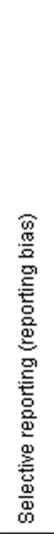 & 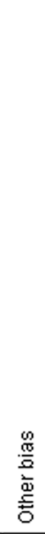 \\
\hline Angiono 2015 & $\theta$ & $\theta$ & $\theta$ & $\odot$ & $?$ & $\odot$ & $\theta$ \\
\hline Chen 2011 & + & $\odot$ & $\theta$ & $\theta$ & + & + & $?$ \\
\hline Choi 2013 & $\theta$ & $\theta$ & $\theta$ & $\Theta$ & $?$ & $?$ & $\theta$ \\
\hline Chung 2015 & + & + & + & $\odot$ & + & + & + \\
\hline Eom 2013 & $\theta$ & $\theta$ & $\theta$ & $\Theta$ & $\odot$ & + & $?$ \\
\hline Fanfani 2012 & $\theta$ & - & $\theta$ & - & $?$ & $?$ & - \\
\hline Fridman 2015 & 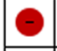 & - & $\theta$ & $\Theta$ & $?$ & $?$ & $\theta$ \\
\hline Hong 2014 & $\theta$ & $\theta$ & 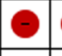 & $\Theta$ & $?$ & - & $?$ \\
\hline Ichikawa 2011 & - & $\theta$ & $\theta$ & $\theta$ & + & $\theta$ & $?$ \\
\hline Jung 2011 & $\odot$ & + & $\theta$ & $\theta$ & $?$ & + & + \\
\hline Jung 2011a & O & $\theta$ & $\theta$ & $\Theta$ & $?$ & $?$ & O \\
\hline Kim 2010 & $\theta$ & $\theta$ & $\theta$ & $\Theta$ & $?$ & $?$ & $?$ \\
\hline Kim SM 2015 & . & $\theta$ & $\theta$ & $\Theta$ & $?$ & $?$ & $?$ \\
\hline Kim TJ 2015 & 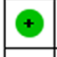 & $\odot$ & $\theta$ & $\Theta$ & + & + & + \\
\hline Koyanagi 2011 & $\theta$ & $\theta$ & $\theta$ & ○ & + & $\theta$ & $\theta$ \\
\hline Lee 2011 & C & $\theta$ & $\theta$ & - & + & O & ? \\
\hline Lee 2011a & $\theta$ & - & \begin{tabular}{|l|}
$?$ \\
\end{tabular} & $\Theta$ & + & + & $?$ \\
\hline Lee 2015 & 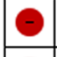 & - & $\theta$ & $\odot$ & $?$ & $?$ & + \\
\hline Li 2012 & $\theta$ & $\theta$ & $?$ & $?$ & + & $\odot$ & $?$ \\
\hline Park 2015 & 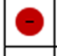 & 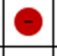 & 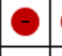 & $\odot$ & $?$ & $?$ & - \\
\hline Song 2013 & 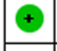 & + & $\theta$ & $?$ & + & $\odot$ & $\odot$ \\
\hline Wang 2012 & | & $\theta$ & $\theta$ & $\odot$ & $?$ & $\theta$ & + \\
\hline Yim 2010 & 9 & $\theta$ & $\theta$ & $\odot$ & $?$ & $?$ & $\ominus$ \\
\hline
\end{tabular}




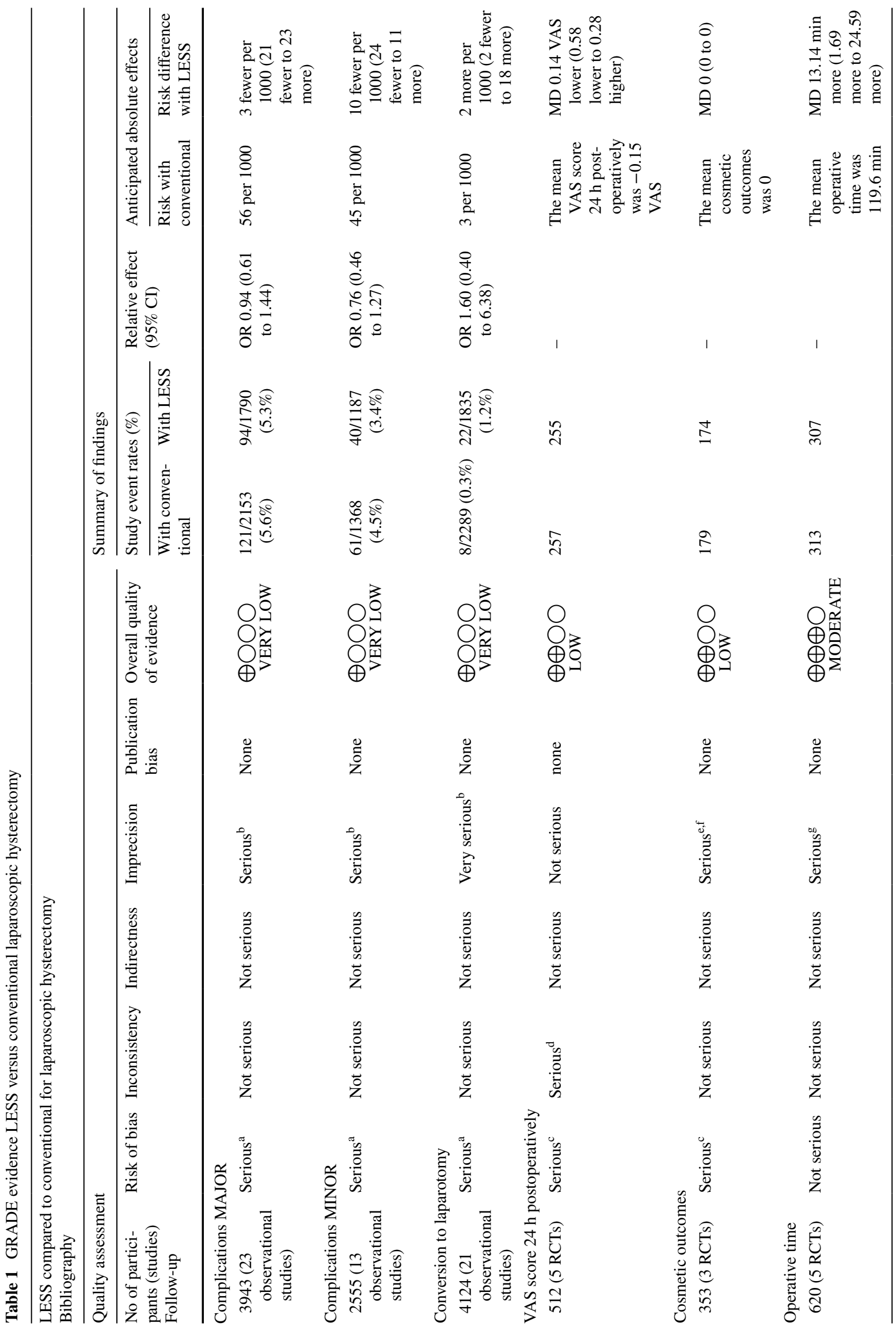




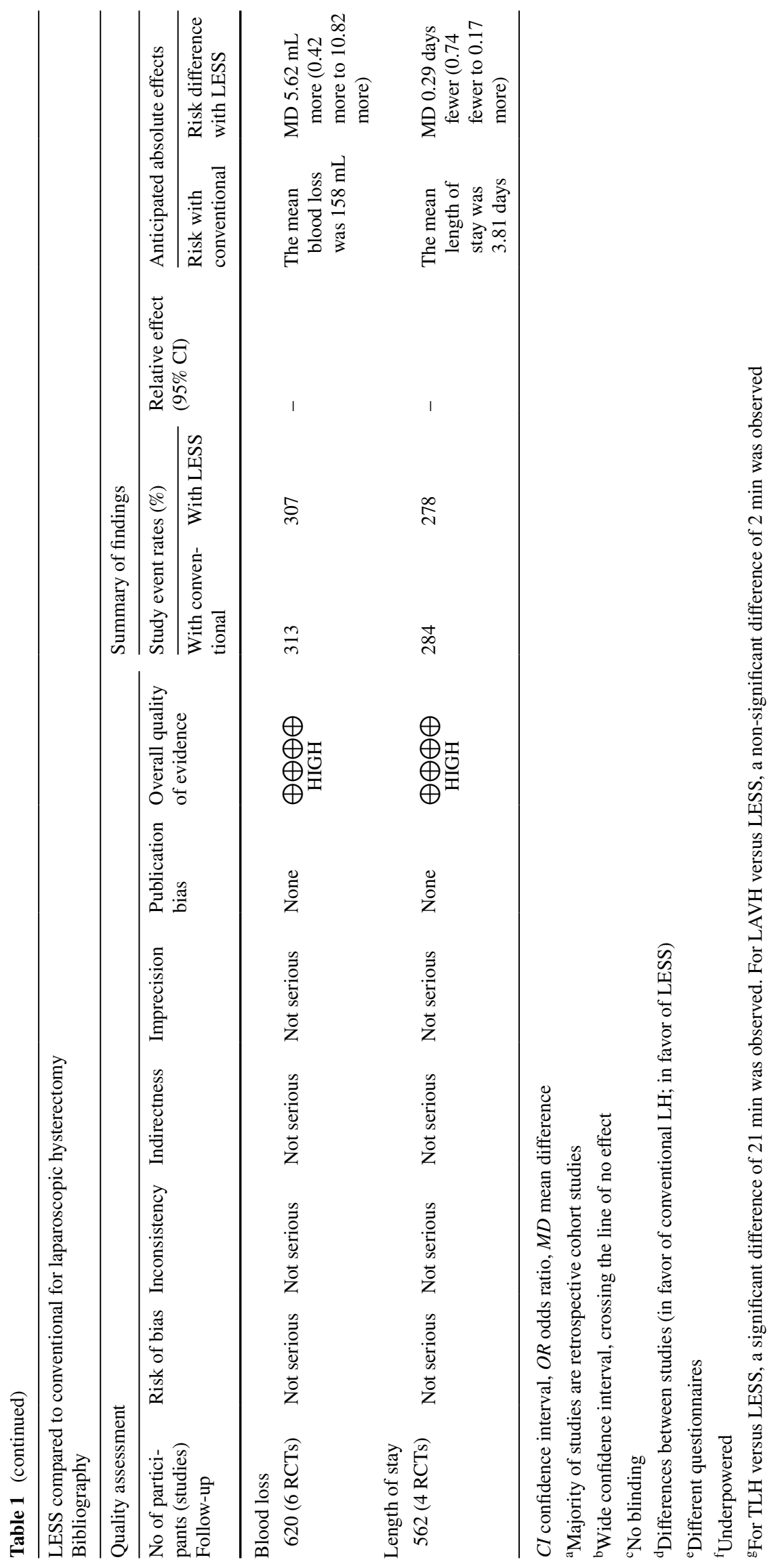


a

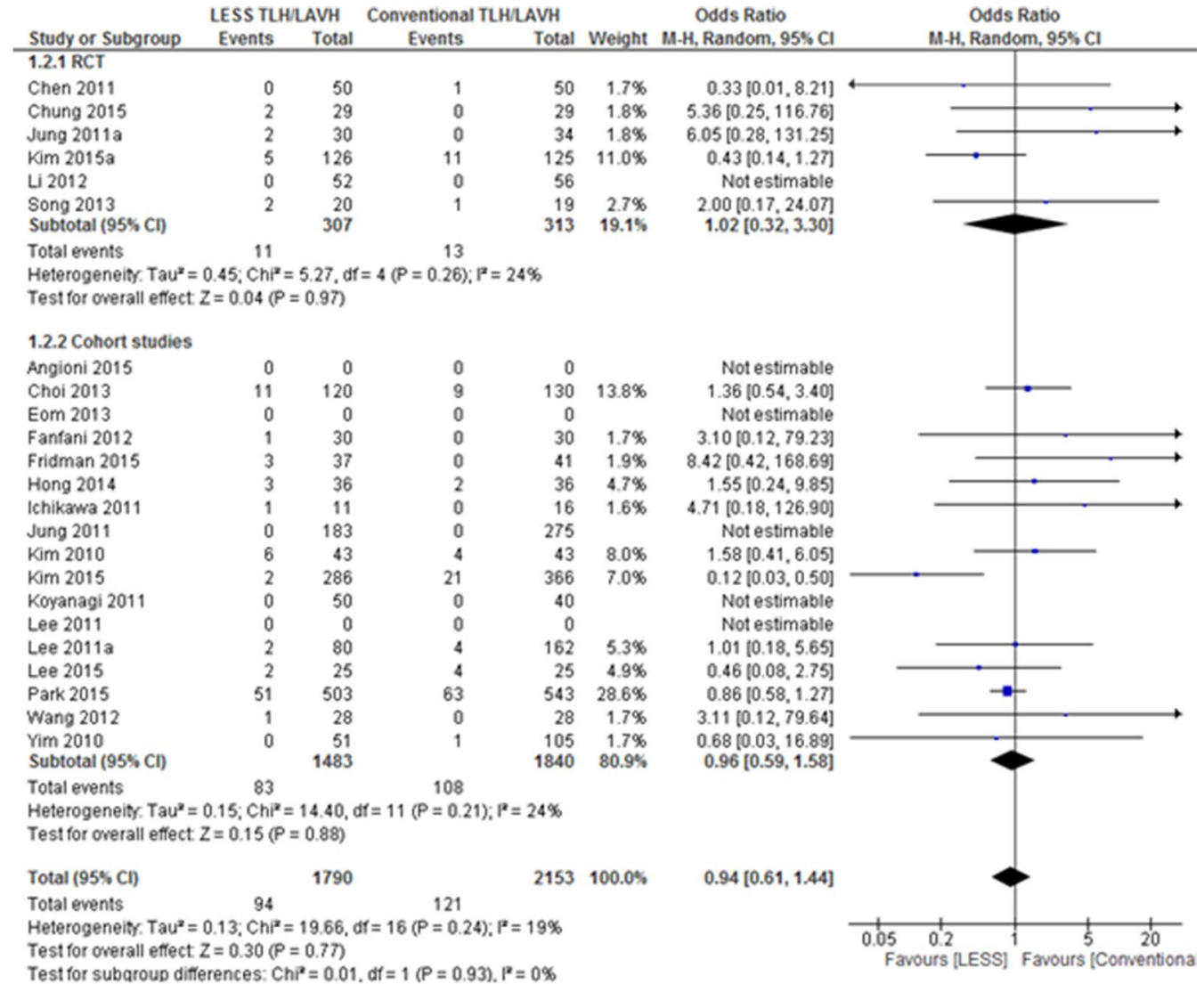

b

LESS Conventional Odds Ratio

Study or Subgroup Events Total Events Total Weight M-H, Random, 95\% Cl MA-H, Random, 95\% Cl

2.1.1 RCT

Chen 2011

Jung 2011a

Kim 2015a

Li 2012

Subtotal $(95 \% \mathrm{Cl})$

Total events

$\begin{array}{lllll}0 & 50 & 1 & 50 & 2.4 \%\end{array}$

930

$1 \quad 126$

$3 \quad 3411.1 \%$

$4 \quad 125 \quad 5.0 \%$

Heterogeneify Tau ${ }^{2}=0.92 ; h^{2}=7.17 . d f=3(P=0.07): P^{2}=58 \%$

Test for overall effect $Z=0.21(P=0.83)$

2.1.2 Cohort studies

Angioni 2015

Choi 2013

Fanfani 2012

Hong 2014

Koyanagi 2011

Lee 2011

Park 2015

Wang 2012

Yim 2010

Subtotal $(95 \% \mathrm{Cl})$

Total events

Heterogeneity: Tau $^{2}=0.00 ;$ Chi $^{2}=4.91$, df $=7(P=0.67) ; P^{2}=0 \%$

Test for overall effect $Z=1.21(P=0.23)$

$\begin{array}{lllll}\text { Total }(95 \% \mathrm{Cl}) & 1187 & 1368 & 100.0 \% & 0.76[0.46,1.27]\end{array}$

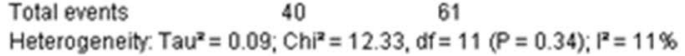

Test for overall effect $Z=1.03(P=0.30)$

Test for subaroup differences: $\mathrm{Ch}^{2}=0.13 . \mathrm{df}=1(\mathrm{P}=0.72), \mathrm{I}^{2}=0 \%$

Odds Ratio

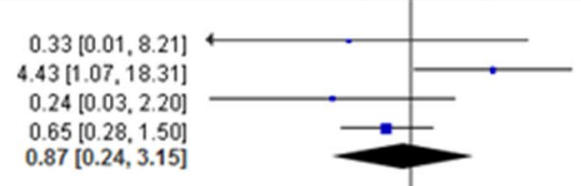

$87[0.24,3.15]$

$0.97[0.06,16.19]$

$1.46[0.32,6.66]$

$1.56[0.24,10.09]$

$0.56[0.12,2.56]$

Not estimable

$0.67[0.07,6.55]$

$0.12[0.01,0.94]$

$0.64[0.10,4.17]$

$0.48[0.10,2.37]$

$0.67[0.35,1.28]$

4
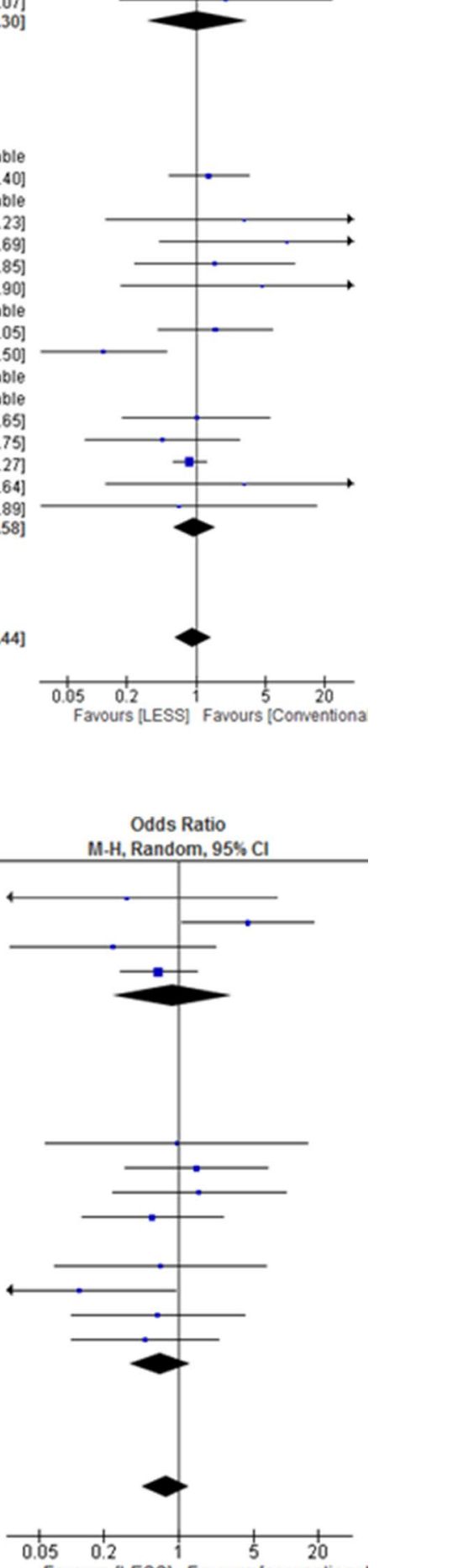

Favours [LESS] Favours [conventional

Fig. 3 Meta-analysis of complications LESS versus conventional laparoscopic hysterectomy 


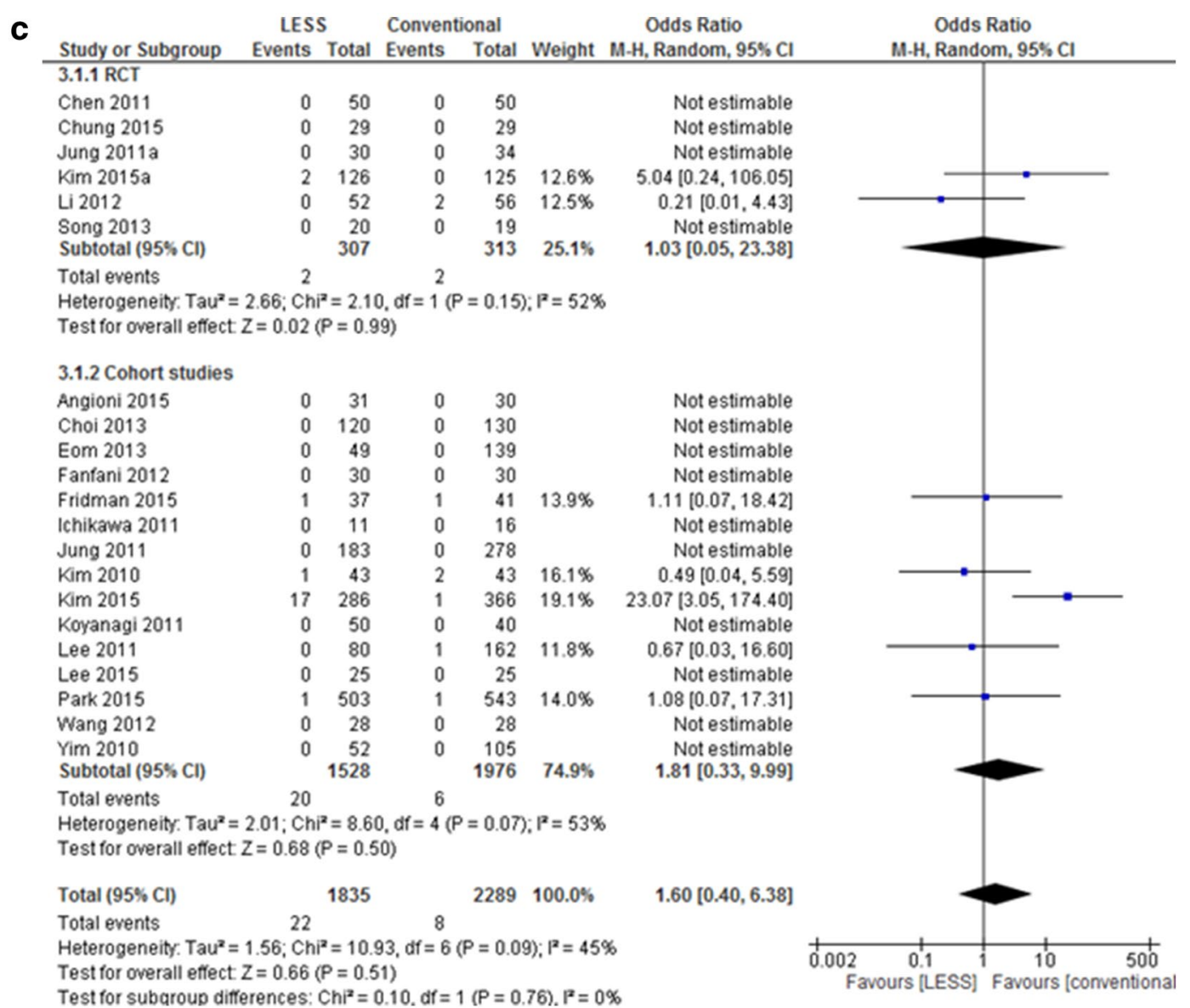

Fig. 3 (continued)

on pain relief and their results were also in favor of the single-port surgery [33]. Finally, Lee et al. [25], Kim et al. 41], and Song et al. [39] showed no difference in analgesic use between the two groups.

Three studies reported on cosmetic results [21, 39, 41], and two used the validated Body Image Questionnaire at 1, 4, and 24 week postoperative. Patients in the LESS group were significantly more satisfied with their scars and had higher satisfaction with their own body at the three measured moments. Kim et al. studied the scar satisfaction using the patient and observer scar assessment scale (POSAS) 1 week and 2 months after surgery and showed no difference between the single-site group and the multi-port one. Li et al. studied patient satisfaction and demonstrated a higher patient satisfaction rate in the single-port group, although it was unclear which questionnaire was used [26]. Lee et al. compared the sexual function of premenopausal women by using the female sexual function index and showed no difference between women that underwent LESS compared to LAVH [36].

\section{Secondary outcomes}

The operative time was significantly longer in the singleport group compared to the multi-port group (20 studies, MD $11.3 \min (5.45-17.17), I^{2}=89 \%$, Fig. 5a). When comparing separately TLH and LAVH, a significant difference of 21 min was seen in favor of the TLH group, compared to a non-significant difference of 2 min after LAVH (data not shown). No difference was seen for the intraoperative blood loss (19 studies, MD $1 \mathrm{~mL}(-6.03,-7.81), I^{2}=27 \%$, Fig. $5 b)$. For the length of hospital stay, a small significant difference was seen (15 studies, MD $-0.22(-0.43,-0.01)$, $I^{2}=86 \%$, Fig. 5c). This difference was not seen when looking separately at the RCTs and cohort studies.

\section{Discussion}

\section{Main findings}

In this systematic review, we evaluated the safety and effectiveness of LESS hysterectomy compared to the 


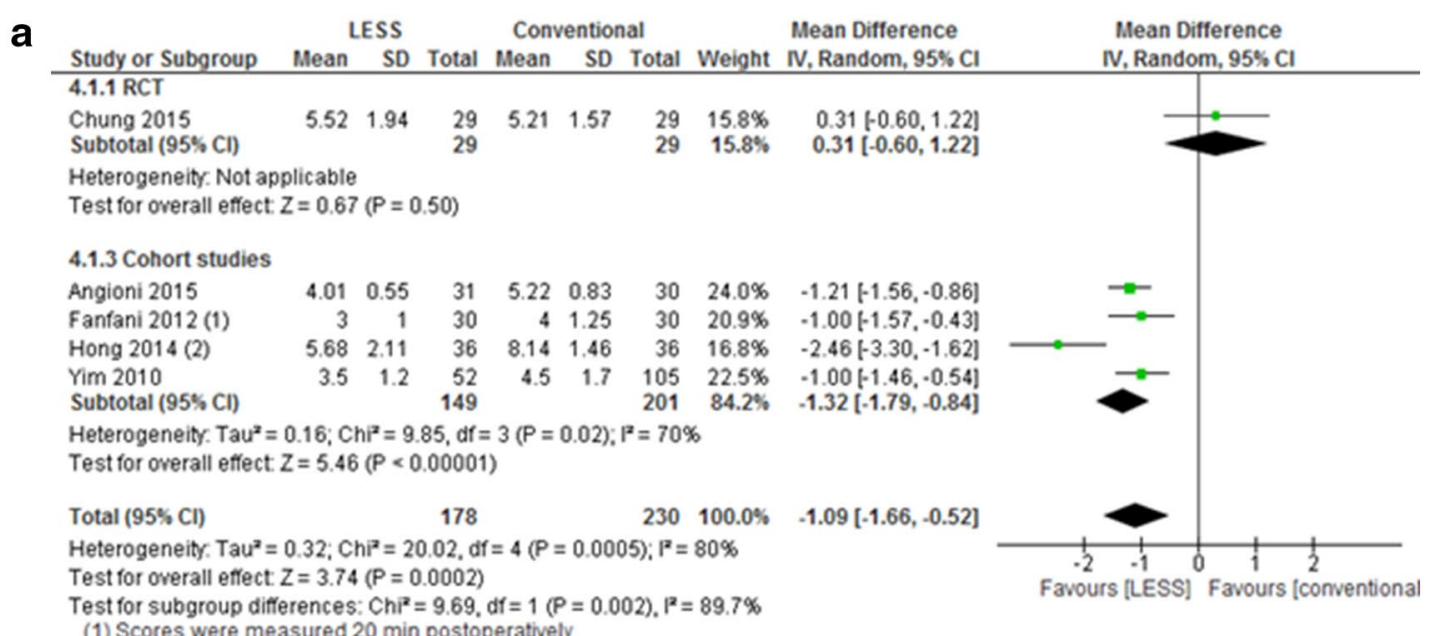

(1) Scores were measured 20 min postoperatively

(2) Direct was defined as direct after surgery up to 2 hours

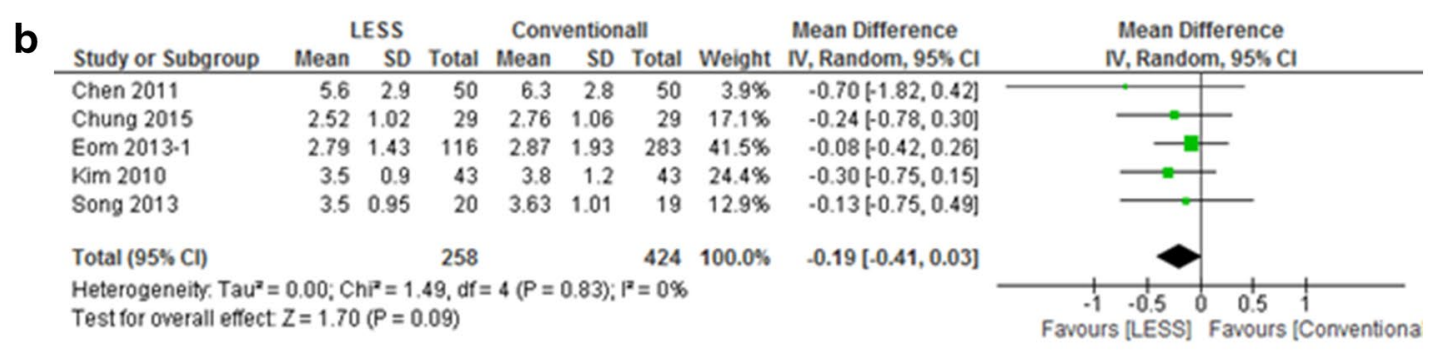

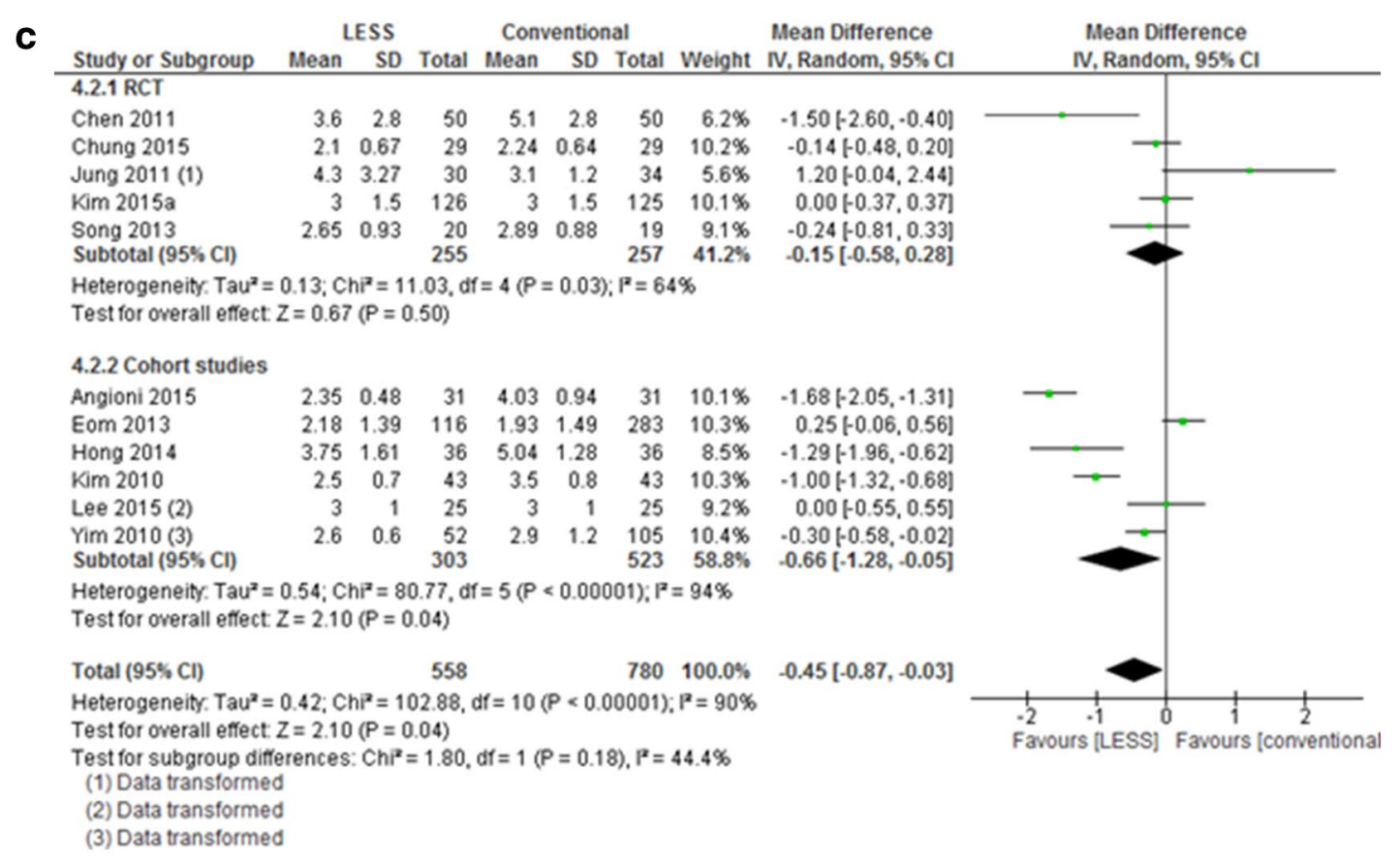

Fig. 4 Meta-analysis of pain scores LESS versus conventional laparoscopic hysterectomy

conventional laparoscopic hysterectomy (TLH and LAVH). Twenty-three studies on LESS versus conventional hysterectomy showed no differences for safety with very low quality evidence. Concerning effectiveness, very low quality evidence indicated no difference for the risk of conversion to laparotomy in the LESS group compared to TLH 
Fig. 5 Meta-analysis of surgical outcomes from LESS versus conventional laparoscopic hysterectomy (operative time, blood loss, and length of stay) a

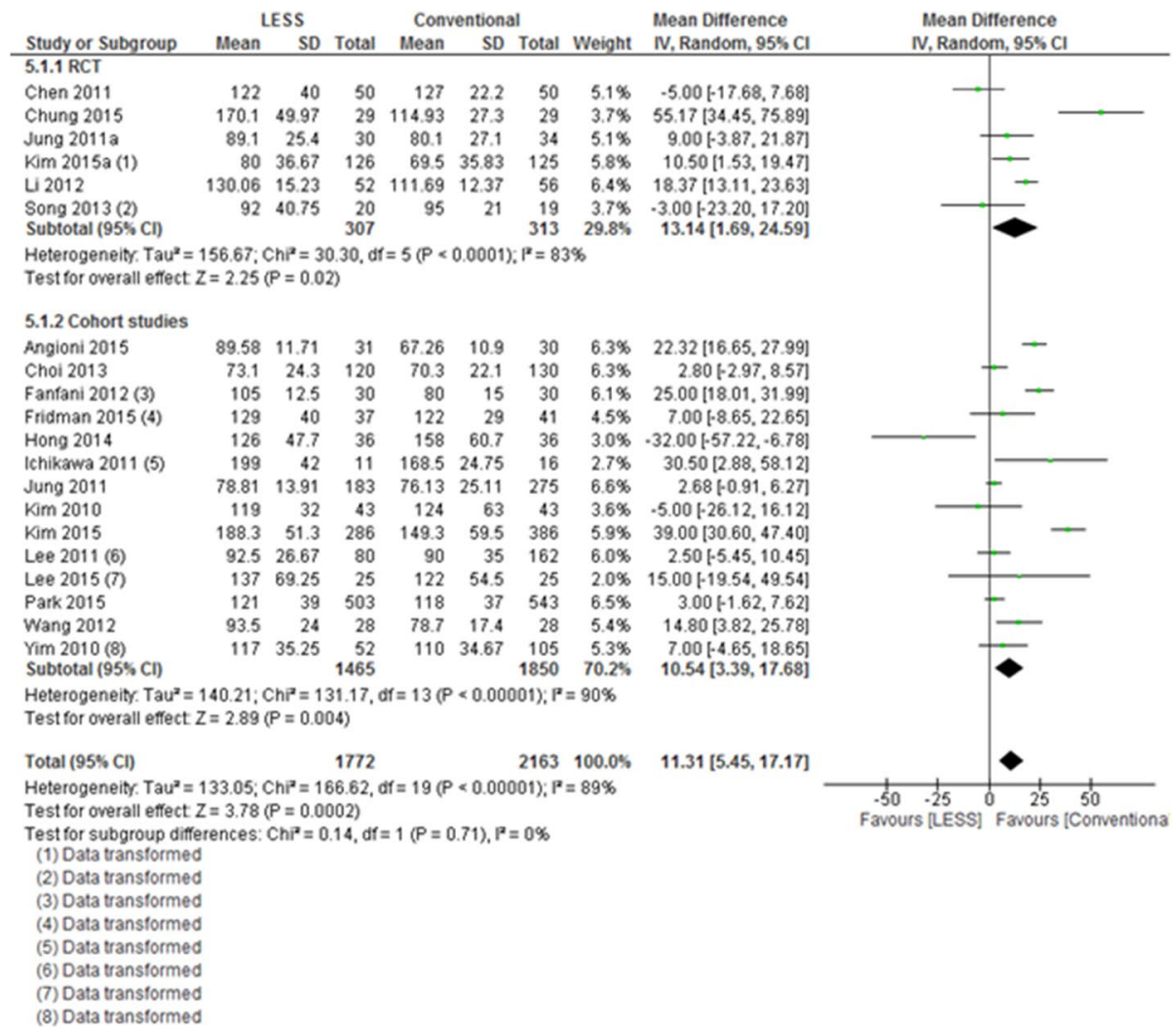


Fig. 5 (continued)

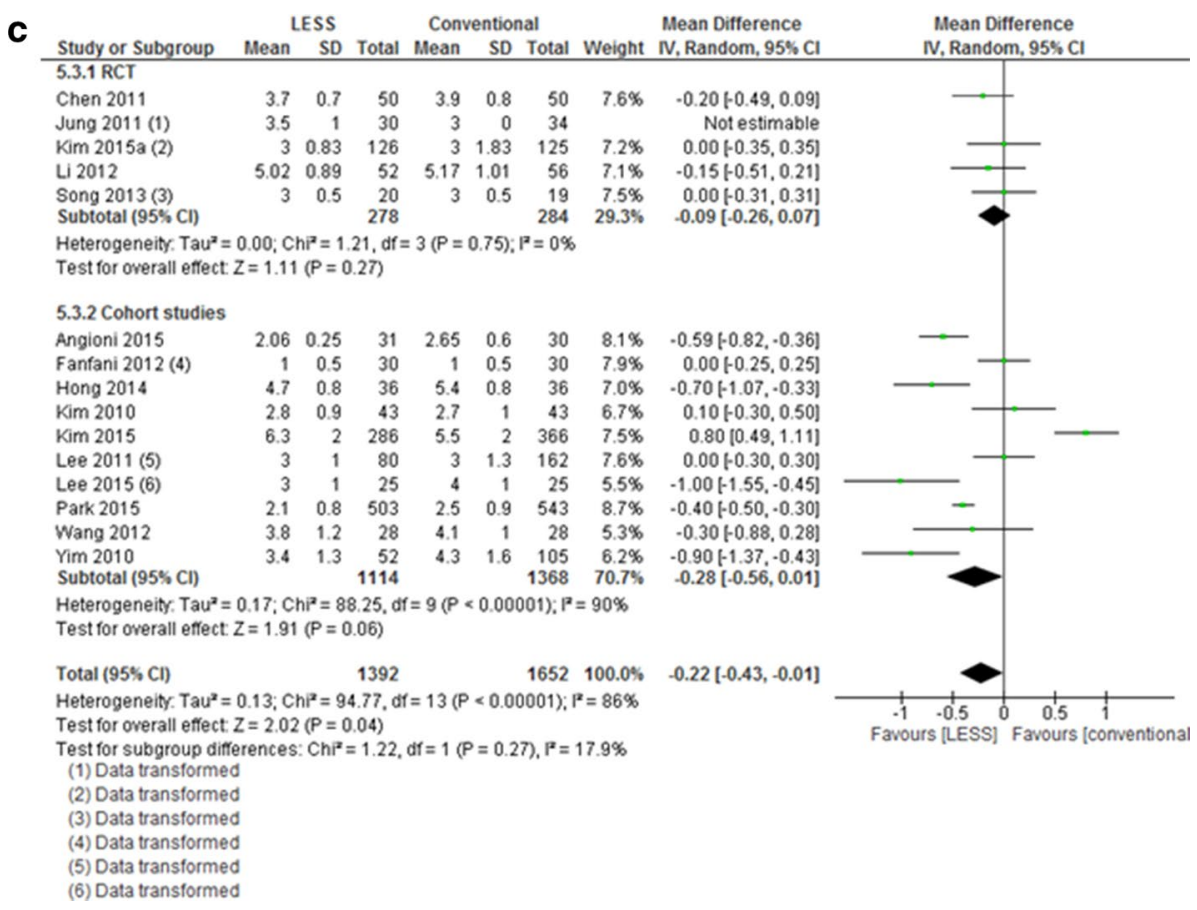

and LAVH. In 3.5\%, the LESS approach failed as an additional port was needed. For postoperative pain, low quality of evidence indicated a lower VAS score of 1.09 and 0.45 , respectively, directly and $24 \mathrm{~h}$ after LESS hysterectomy, though with substantial statistical heterogeneity. Two out of three studies with low-quality evidence indicated a better cosmetic outcome after LESS versus conventional hysterectomy. A major shortcoming in these studies is the lack of a pre-operative assessment. Without a pre-operative assessment, it remains unclear whether there were any differences between the groups prior to their surgery. The third study, an RCT showed no difference with respect to scar satisfaction.

\section{Strengths and limitations}

Though there are some RCTs available comparing LESS to conventional hysterectomy, we decided to include other comparative study designs as well. The inclusion of nonRCT designs results in less homogenous groups, but when outcomes of interest are infrequent (e.g., conversion-tolaparotomy risk, complication risks); RCTs are rarely large and lengthy enough to measure infrequent outcomes accurately. Cohort studies facilitate a larger study population and adequate power to identify significant differences. Therefore, the inclusion of study designs other than RCTs can be seen as a limitation but also as strength. In addition, to limit bias, we performed sensitivity analysis for the study design for the meta-analysis. Another strength of this review is the assessment of the quality of evidence using
GRADE methodology. We believe that the use of GRADE results in additional clinical value of this review: GRADE optimizes the presentation of evidence for clinical practice. The results of this systematic review are strengthened through the findings of other reviews published on the subject that as well found no significant difference in the frequency of perioperative complications and postoperative pain scores [8, 9, 43]. Though, other reviews described a higher rate of 'failures' in the LESS group. These studies defined 'failure' as the need to convert to laparotomy and/ or to add an extra port, without differentiating. We found that in $3.5 \%$ of the LESS procedures, an additional port was needed compared to $<1 \%$ in the conventional procedures.

\section{Interpretation}

The feasibility of LESS surgery for benign gynecologic procedures seems proven [8,9]. The meta-analyses in this review showed no significant differences in complication and conversion-rate to laparotomy between LESS and conventional hysterectomy. Without substantial statistical heterogeneity, we consider these findings reliable. Besides complication risk, the pain experienced after surgery is an important consideration and usually an important argument in favor of LESS. Though, we did not find any clinically significant differences in postoperative pain. Directly and $24 \mathrm{~h}$ after LESS hysterectomy, a significant lower VAS score was observed. This difference was not observed when analyzing only the RCTs. Furthermore, the mean difference did not exceed 1.09 and studies have shown that a 
mean difference of 2 points on a 10-point scale should be considered as clinically relevant [44]. In addition, it cannot be excluded that enrolled patients in the study are biased with respect to their pain outcomes as, except in one study, the included patients were not blinded to the type of surgery. One single randomized controlled trial applied accurate blinding [23]: patients and anesthesiology staff who measured the postoperative pain scores did not know which type of approached had been performed and similar pain scores were found. Cosmetic outcomes are also suggested as important improvement in the single-site approach but surprisingly few studies on LESS hysterectomy reported on this topic [21, 39, 41]. We judged the assessment in the two studies on patient satisfaction insufficient, since baseline assessment of body image and cosmetic satisfaction was not performed. The largest RCT published so far for hysterectomy reported no significant differences regarding scar satisfaction between the LESS and 'conventional' hysterectomy group. When looking at studies published in other fields than benign gynecology, inconsistent results are found for the self-scar rating in patients who underwent LESS or conventional laparoscopic surgery [45-47]. In Tuschy et al. patients who underwent conventional gynecological laparoscopy were asked which scar they would prefer to eliminate, and for most patients, it was the umbilical one [48]. In the study by Bush et al. patients were asked their aesthetic preference regarding scars, and no differences were observed between the single-site and conventional incisions [6]. In LESS surgery, higher forces are applied on the umbilical port during tissue handling and irreversible umbilical deformation has been described [29]. It is also suggested that LESS would lead to a higher risk of port herniation as the opening of the umbilical port is larger [49-51]. Though, this could not be confirmed in the current literature, as within the short study follow-up, only one case of port herniation was reported [31].

Evaluating the secondary surgical outcomes, a notable finding is the increased operative time found in the LESS versus conventional hysterectomy group: an overall mean difference of 11 min was observed, though with substantial heterogeneity. For the TLH, the mean difference was $21 \mathrm{~min}$, whereas for the LAVH, a non-significant difference of $2 \mathrm{~min}$ was observed. The reason for the prolonged operative time during TLH is most probably related to the difference in surgical experience. For the LAVH, it makes sense that the operative time was similar as a large part of the LESS and conventional procedure is performed vaginally, thus using exactly similar techniques. It is well known that LESS surgery is technically more challenging $[8,9,43]$ and studies reporting on the learning curve in LESS have suggested that sufficient skills are acquired after 10 to 15 [3] up to 40 cases [52], especially when surgeons are already well-trained in laparoscopy. In five studies included in this review, the surgical experience of the surgeons was not described [13, 28, 30, 35, 38]. In the other included studies, the experience of surgeons was defined by terms, such as 'very experienced', 'senior surgeon', or by the number of laparoscopic and/or LESS surgeries performed in one's career. Hence, it is difficult to interpret the impact of the skills on the outcomes. It is noteworthy mentioning that we found substantial differences in baseline characteristics between compared groups in the non-randomized studies (uterine weight [20, 21, 28], age [20], BMI [31], previous surgeries, and co-morbidities [28, 38]). This could be explained by the surgeon's specific selection when performing a new technique in a non-randomized setting. Yet, an increased uterine weight, a high BMI, and/or previous surgical interventions are known to directly influence surgical outcomes [53] and this could lead to an overestimation of effectiveness, safety, and secondary outcomes (e.g., operative time, blood loss) for LESS outcomes. In addition, it should also be taken into account that 20 of the 23 studies originated from Asian, and therefore, the impact of Asian demographics should not be underestimated.

Remarkably, none of the included studies has taken the costs of the surgery into account, and currently, it is unknown if the LESS approach is cost effective. Despite the lack of data for LESS versus conventional hysterectomy, it can be reasoned that implementing the LESS technique in a hospital is costly as the conventional instruments do not fit and new instruments need to be purchased.

As seen with previous devices and or techniques [54], implementing new technologies in the medical field is a challenge. In contrast to the introduction of new drugs, the latest techniques and devices are usually implemented in clinical practice without proper systematic evaluation regarding their safety, effectiveness, costs, and benefits. Advantages and disadvantages only become clear with the passage of time and after the implantation phase. Considering this, it is complex to answer the question whether the single-port surgery should be an additional possibility for the minimally invasive surgery. Most of studies in the review were single center and from the same region in the world, where a lot of experienced has already been acquired with the LESS technique. Despite the amount of experience with LESS in these centers, there is still no clear added value.

In conclusion, current evidence shows that the singleport technique for benign hysterectomy (TLH and LAVH) is feasible, safe, and equally effective compared to the conventional technique. Caution is urged when interpreting the results of studies on LESS because the evidence is of lowto-very low quality. Potential benefits are sought in patient satisfaction, cosmetic satisfaction, and postoperative pain, but the small differences for these outcomes appear not to be of clinical relevance. Furthermore, surgeons and patients 
should be aware that in up to $3.5 \%$ of LESS hysterectomies an additional port is required resulting in failure of the "single site" approach and affecting the less invasive purpose. As no clinically relevant advantages were identified, and no data on cost effectiveness were available, there are currently no solid arguments to implement the single-port technique worldwide.

Author contributions EMS: protocol/project development, data collection or management, data analysis, manuscript writing/editing. CFC: protocol/project development, data collection or management, data analysis, manuscript writing/editing. MMT: protocol/project development, data collection or management, data analysis, manuscript writing/editing. JWS: developed literature search strategy, data collection or management, manuscript writing/editing. FWJ: protocol/ project development, manuscript writing/editing.

\section{Compliance with ethical standards}

Funding This study was not funded.

Conflict of interest Evelien Sandberg has received a grant from the Bronovo Research Fund (Bronovo Hospital, The Hague, The Netherlands). The other authors declare that they have no conflict of interest.

Ethical approval This article does not contain any studies with human participants or animals performed by any of the authors.

Open Access This article is distributed under the terms of the Creative Commons Attribution 4.0 International License (http:// creativecommons.org/licenses/by/4.0/), which permits unrestricted use, distribution, and reproduction in any medium, provided you give appropriate credit to the original author(s) and the source, provide a link to the Creative Commons license, and indicate if changes were made.

\section{References}

1. Aarts JW, Nieboer TE, Johnson N et al (2015) Surgical approach to hysterectomy for benign gynaecological disease. Cochrane Database Syst Rev 8:CD003677

2. Fader AN, Cohen S, Escobar PF, Gunderson C (2010) Laparoendoscopic single-site surgery in gynecology. Curr Opin Obstet Gynecol 22:331-338

3. Escobar PF, Starks D, Fader AN, Catenacci M, Falcone T (2010) Laparoendoscopic single-site and natural orifice surgery in gynecology. Fertil Steril 94:2497-2502

4. Mencaglia L, Mereu L, Carri G et al (2013) Single port entry are there any advantages? Best Pract Res Clin Obstet Gynaecol 27:441-455

5. Fanfani F, Rossitto C, Gagliardi ML et al (2012) Total laparoendoscopic single-site surgery (LESS) hysterectomy in low-risk early endometrial cancer: a pilot study. Surg Endosc 26:41-46

6. Bush AJ, Morris SN, Millham FH, Isaacson KB (2011) Women's preferences for minimally invasive incisions. J Minim Invasive Gynecol 18:640-643

7. Pelosi MA, Pelosi MA III (1991) Laparoscopic hysterectomy with bilateral salpingo-oophorectomy using a single umbilical puncture. N J Med 88:721-726
8. Song T, Kim ML, Jung YW, Yoon BS, Joo WD, Seong SJ (2013) Laparoendoscopic single-site versus conventional laparoscopic gynecologic surgery: a metaanalysis of randomized controlled trials. Am J Obstet Gynecol 209:317-319

9. Yang L, Gao J, Zeng L, Weng Z, Luo S (2015) Systematic review and meta-analysis of single-port versus conventional laparoscopic hysterectomy. Int J Gynaecol Obstet 133:9-16

10. Driessen SR, Baden NL, van Zwet EW, Twijnstra AR, Jansen FW (2015) Trends in the implementation of advanced minimally invasive gynecologic surgical procedures in the Netherlands. $\mathrm{J}$ Minim Invasive Gynecol 22:642-647

11. Wright JD, Herzog TJ, Tsui J et al (2013) Nationwide trends in the performance of inpatient hysterectomy in the United States. Obstet Gynecol 122:233-241

12. Makinen J, Brummer T, Jalkanen J et al (2013) Ten years of progress-improved hysterectomy outcomes in Finland 1996-2006: a longitudinal observation study. BMJ Open 3:e003169

13. Kim SM, Park EK, Jeung IC, Kim CJ, Lee YS (2015) Abdominal, multi-port and single-port total laparoscopic hysterectomy: eleven-year trends comparison of surgical outcomes complications of 936 cases. Arch Gynecol Obstet 291:1313-1319

14. Stroup DF, Berlin JA, Morton SC et al (2000) Meta-analysis of observational studies in epidemiology: a proposal for reporting. Meta-analysis Of Observational Studies in Epidemiology (MOOSE) group. JAMA 283:2008-2012

15. Complication registration system of the Dutch Society of Obstetrics and Gynecology. http://www.nvog.nl/vakinformatie/ Pati\%C3\%ABntveiligheid/Complicatieregistratie/default.aspx. Accessed 25 Mar 2017

16. Joyce CR, Zutshi DW, Hrubes V, Mason RM (1975) Comparison of fixed interval and visual analogue scales for rating chronic pain. Eur J Clin Pharmacol 8:415-420

17. Guyatt GH, Oxman AD, Vist G et al (2011) GRADE guidelines: 4. Rating the quality of evidence-study limitations (risk of bias). J Clin Epidemiol 64:407-415

18. Guyatt GH, Oxman AD, Schunemann HJ, Tugwell P, Knottnerus A (2011) GRADE guidelines: a new series of articles in the. J Clin Epidemiol 64:380-382

19. Hozo SP, Djulbegovic B, Hozo I (2005) Estimating the mean and variance from the median, range, and the size of a sample. BMC Med Res Methodol 5:13

20. Fridman D, Saraf S, Homel P, Wagner J (2015) Single-incision laparoscopy as the primary approach to benign hysterectomies: a single-surgeon, single-year-experience with a retrospective control. J Gynecol Surg 31:78-82

21. Angioni S, Pontis A, Pisanu A, Mereu L, Roman H (2015) Single-port access subtotal laparoscopic hysterectomy: a prospective case-control study. J Minim Invasive Gynecol 22:807-812

22. Fanfani F, Fagotti A, Rossitto C et al (2012) Laparoscopic, minilaparoscopic and single-port hysterectomy: perioperative outcomes. Surg Endosc 26:3592-3596

23. Chung JH, Baek JM, Chung K et al (2015) A comparison of postoperative pain after transumbilical single-port access and conventional three-port total laparoscopic hysterectomy: a randomized controlled trial. Acta Obstet Gynecol Scand 94:1290-1296

24. Jung YW, Lee M, Yim GW et al (2011) A randomized prospective study of single-port and four-port approaches for hysterectomy in terms of postoperative pain. Surg Endosc 25:2462-2469

25. Lee J, Kim S, Nam EJ, Hwang SM, Kim YT, Kim SW (2015) Single-port access versus conventional multi-port access total laparoscopic hysterectomy for very large uterus. Obstet Gynecol Sci 58:239-245

26. Li M, Han Y, Feng YC (2012) Single-port laparoscopic hysterectomy versus conventional laparoscopic hysterectomy: a prospective randomized trial. J Int Med Res 40:701-708 
27. Wang T, Chong GO, Park NY, Hong DG, Lee YS (2012) Comparison study of single-port (Octoport) and four-port total laparoscopic hysterectomy. Eur J Obstet Gynecol Reprod Biol $161: 215-218$

28. Yim GW, Jung YW, Paek J et al (2010) Transumbilical singleport access versus conventional total laparoscopic hysterectomy: surgical outcomes. Am J Obstet Gynecol 203:26

29. Ichikawa M, Akira S, Mine K et al (2011) Evaluation of laparoendoscopic single-site gynecologic surgery with a multitrocar access system. J Nippon Med Sch 78:235-240

30. Chen YJ, Wang PH, Ocampo EJ, Twu NF, Yen MS, Chao KC (2011) Single-port compared with conventional laparoscopicassisted vaginal hysterectomy: a randomized controlled trial. Obstet Gynecol 117:906-912

31. Choi YS, Park JN, Oh YS, Sin KS, Choi J, Eun DS (2013) Single-port vs. conventional multi-port access laparoscopy-assisted vaginal hysterectomy: comparison of surgical outcomes and complications. Eur J Obstet Gynecol Reprod Biol 169:366-369

32. Eom JM, Choi JS, Choi WJ, Kim YH, Lee JH (2013) Does single-port laparoscopic surgery reduce postoperative pain in women with benign gynecologic disease? J Laparoendosc Adv Surg Tech A 23:999-1005

33. Hong M-K, Wang J-H, Chu T-Y, Ding D-C (2014) Laparoendoscopic single-site hysterectomy with Ligasure is better than conventional laparoscopic assisted vaginal hysterectomy. Gynecol Minim Invasive Ther 3:78-81

34. Jung MH, Lee BY (2011) Transumbilical single-port laparoscopic-assisted vaginal hysterectomy via 12-mm trocar incision site. J Laparoendosc Adv Surg Tech A 21:599-602

35. Kim TJ, Lee YY, Cha HH et al (2010) Single-port-access laparoscopic-assisted vaginal hysterectomy versus conventional laparoscopic-assisted vaginal hysterectomy: a comparison of perioperative outcomes. Surg Endosc 24:2248-2252

36. Lee JH, Choi JS, Hong JH, Joo KJ, Kim BY (2011) Does conventional or single port laparoscopically assisted vaginal hysterectomy affect female sexual function? Acta Obstet Gynecol Scand 90:1410-1415

37. Lee JH, Choi JS, Jeon SW, Son CE, Hong JH, Bae JW (2011) A prospective comparison of single-port laparoscopically assisted vaginal hysterectomy using transumbilical GelPort access and multiport laparoscopically assisted vaginal hysterectomy. Eur J Obstet Gynecol Reprod Biol 158:294-297

38. Park JY, Nho J, Cho IJ et al (2015) Laparoendoscopic singlesite versus conventional laparoscopic-assisted vaginal hysterectomy for benign or pre-invasive uterine disease. Surg Endosc 29:890-897

39. Song T, Cho J, Kim TJ et al (2013) Cosmetic outcomes of laparoendoscopic single-site hysterectomy compared with multi-port surgery: randomized controlled trial. J Minim Invasive Gynecol $20: 460-467$

40. Koyanagi T, Motomura S (2011) Single-incision laparoscopically assisted vaginal hysterectomy: operative outcomes and its learning curve. Exp Ther Med 2:867-871

41. Kim TJ, Shin SJ, Kim TH et al (2015) Multi-institution, prospective, randomized trial to compare the success rates of single-port versus multiport laparoscopic hysterectomy for the treatment of uterine myoma or adenomyosis. J Minim Invasive Gynecol 22:785-791

42. Koyanagi T, Motomura S (2011) Transumbilical single-incision laparoscopic surgery: application to laparoscopically assisted vaginal hysterectomy. Arch Gynecol Obstet 283:305-309

43. Pontis A, Sedda F, Mereu L et al (2016) Review and metaanalysis of prospective randomized controlled trials (RCTs) comparing laparo-endoscopic single site and multiport laparoscopy in gynecologic operative procedures. Arch Gynecol Obstet 294:567-577

44. Giraudeau B, Rozenberg S, Valat JP (2004) Assessment of the clinically relevant change in pain for patients with sciatica. Ann Rheum Dis 63:1180-1181

45. Song T, Park JY, Kim TJ et al (2015) A prospective comparative study of cosmetic satisfaction for three different surgical approaches. Eur J Obstet Gynecol Reprod Biol 190:48-51

46. Park SK, Olweny EO, Best SL, Tracy CR, Mir SA, Cadeddu JA (2011) Patient-reported body image and cosmesis outcomes following kidney surgery: comparison of laparoendoscopic singlesite, laparoscopic, and open surgery. Eur Urol 60:1097-1104

47. Eom JM, Ko JH, Choi JS, Hong JH, Lee JH (2013) A comparative cross-sectional study on cosmetic outcomes after single port or conventional laparoscopic surgery. Eur J Obstet Gynecol Reprod Biol 167:104-109

48. Tuschy B, Berlit S, Brade J, Sutterlin M, Hornemann A (2013) Gynaecological laparoscopic surgery for benign conditions: do women care about incisions? Eur J Obstet Gynecol Reprod Biol 169:84-87

49. Kadar N, Reich H, Liu CY, Manko GF, Gimpelson R (1993) Incisional hernias after major laparoscopic gynecologic procedures. Am J Obstet Gynecol 168:1493-1495

50. Gunderson CC, Knight J, Ybanez-Morano J et al (2012) The risk of umbilical hernia and other complications with laparoendoscopic single-site surgery. J Minim Invasive Gynecol 19:40-45

51. Mayol J, Garcia-Aguilar J, Ortiz-Oshiro E, De-Diego Carmona JA, Fernandez-Represa JA (1997) Risks of the minimal access approach for laparoscopic surgery: multivariate analysis of morbidity related to umbilical trocar insertion. World J Surg 21:529-533

52. Paek J, Kim SW, Lee SH et al (2011) Learning curve and surgical outcome for single-port access total laparoscopic hysterectomy in 100 consecutive cases. Gynecol Obstet Invest $72: 227-233$

53. Driessen SR, Sandberg EM, la Chapelle CF, Twijnstra AR, Rhemrev JP, Jansen FW. Case-mix variables and predictors for outcomes of laparoscopic hysterectomy: a systematic review. doi:10.1016/j.jmig.2015.11.008

54. Laparoscopic Uterine Power Morcellation in Hysterectomy and Myomectomy: FDA Safety Communication. http://www.fda. gov/medicaldevices/safety/alertsandnotices/ucm393576.htm. Accessed 25 Mar 2017 